\title{
THE FEBRUARY MEETING OF THE SOCIETY
}

The two hundred twenty-seventh regular meeting of the Society was held at Columbia University on Saturday, February 24 , with the usual morning and afternoon sessions. The attendance included the following thirty-five members:

Alexander, Archibald, Barnum, W. J. Berry, Cole, Cowley, Dantzig, Fite, Fry, Gafafer, Glenn, Gronwall, Hausle, Joffe, Kasner, Langman, MacColl, Meder, F. Morley, Mullins, Northcott, Oglesby, Paaswell, Pfeiffer, Reddick, R. G. D. Richardson, Ritt, Seely, Siceloff, Sosnow, Tracey, H. E. Webb, Weisner, Whited, Whittemore.

The Secretary announced the election of the following persons to membership in the Society:

Miss Frances Almira Atwater, Milwaukee-Downer College;

Mr. Heinrich Wilhelm Brinkman, Harvard University;

Professor Robert Ernest Bruce, Boston University;

Professor Joseph Edward Burnam, Simmons College;

Professor Frederick John Dick, Theosophical University;

Mr. Lawrence Murray Graves, University of Chicago;

Mr. Albrecht Wladimir Hanush, Chicago, Ill.;

Mr. Dio Lewis Holl, University of Chicago;

Professor Anna Mayme Howe, Newcomb College;

Professor Emma Louise Konantz, Peking University;

Mr. Bernard Osgood Koopman, Harvard University;

Miss Anna Marguerite Marie Lehr, Bryn Mawr College;

Dr. Earl Emanuel Libman, University of Illinois;

Mr. Robert Bruce Lindsay, Fellow, American-Scandinavian Foundation;

Mr. Renke Gustav Lubben, University of Texas;

Professor Dayton Clarence Miller, Case School of Applied Science;

Mr. Murtach Matthew Sylvester Moriarty, Holyoke, Mass.;

Mr. David Rines, Boston, Mass.;

Limmye Vernon Robinson, Meridian College;

Professor George Wellington Spenceley, Miami University;

Dr. Flora Dobler Sutton, Johns Hopkins University;

Dr. William Phillip Udinski, Texas Agricultural and Mechanical College;

Professor David Locke Webster, Stanford University;

Mr. William Marvin Whyburn, University of Texas;

Miss Constance Wiener, Smith College;

Mr. Walter Wood, Philadelphia, Pa.

Since the Annual Meeting, the following additional members of the London Mathematical Society have entered the American Mathematical Society under the reciprocity agreement:

Mr. John Brill, London;

Professor Horatio Scott Carslaw, University of Sydney; 
Professor Robert William Genese, Hastings;

Mr. William Percy Dartrey MacMahon, English High School, Constantinople;

Professor John Raymond Wilton, University of Adelaide.

Twenty applications for membership in the Society were received.

The following committees were appointed:-On Arrangements for the Summer Meeting, H. S. White (chairman), G. M. Conwell, E. B. Cowley, L. D. Cummings, and the Secretary; On Policy and Budget, the President (chairman), Treasurer and Secretary, and E. T. Bell, Henry Blumberg, L. P. Eisenhart, E. R. Hedrick, and A. J. Pell; On Endowment, J. L. Coolidge (chairman), G. E. Roosevelt (secretary), Arnold Dresden, G. C. Evans, and Robert Henderson.

Professor E. V. Huntington was appointed representative of the Society in the Division of Physical Sciences of the National Research Council for the period of three years beginning July 1, 1923, as successor to Professor Veblen.

At the morning session of the Society, Ex-President Frank Morley presided, and at the afternoon session, Professor J. K. Whittemore.

Titles and abstracts of the papers read at this meeting follow below. The papers of $\mathrm{Mr}$. Garabedian, Professor Hollcroft, Mr. Murray, Professor Emch, Dr. Walsh, Dr. Nelson, Professor Schwatt, Dr. Rutledge, Professor Lipka, Mr. Wilder, Mr. Rice, and Professor Kakeya were read by title. Miss Whelan's paper was read by Professor Morley. Miss Whelan was introduced by Professor Morley, Dr. Rainich by Dr. Pfeiffer, and Professor Kakeya by Professor Birkhoff.

1. Mr. C. A. Garabedian: Circular plates of constant or variable thickness.

This paper develops a method of series in elasticity, with particular reference to its applications to circular plates of constant or variable thickness. MM. E. and M. Cosserat, in a note in the Comptes Rendus, vol. 146 (1908), show how a method of series, that takes account of the geometric parameters that must enter, will yield results in agreement with classical solutions; their applications are confined to cases of 
constant thickness. In his note on Circular plates of variable thickness, Philosophical Magazine, May, 1922, Professor Birkhoff adopts cylindrical coordinates, with the axis of $z$ along the axis of the plate; he then writes $z=\zeta t$, assumes the displacements developable in power series in $t$, and, by a method of minimizing the energy integral, obtains leading terms when the thickness is variable. The present author uses power series in $t$, but takes as his starting point the fundamental equations of elasticity. This results in a direct method of series, applicable to cases of variable as well as constant thickness. The method is capable of extension, and affords a solution of a problem which has interested elasticians since Poisson and Cauchy.

2. Professor J. F. Ritt: Permutable rational functions.

This paper investigates the circumstances under which two rational functions, $\varphi(x)$ and $\psi(x)$, are such that $\varphi[\psi(x)]=$ $\psi[\varphi(x)]$. A detailed statement of results has appeared in the Comptes Rendus for January 8, 1923.

3. Professor T. R. Hollcroft: The maximum number of cusps of a space curve.

From a formula for the maximum genus of a space curve of given order $n$ due to Noether, the minimum number of apparent double points is found. Consider the curve projected on a plane from a point of the nodal curve of its developable, or, for $n$ large, from a triple point of this curve. From any point of the nodal curve, two apparent double points project into cusps and from a triple point of this curve, three. The remaining apparent double points project into nodes of the plane curve. The maximum number of cusps that may be added to the singularities of this plane curve is the number that may result from the projection of cusps of the space curve. This gives formulas for the maximum number of cusps of a space curve of given order $n$ lying on a nonsingular surface of given order $\mu$. For $\mu=2$ the maximum number of cusps for a given $n$ is given by formulas involving only $n$, and this theorem follows: Every proper space curve with the maximum number of cusps for a given order lies on a quadric surface.

4. Miss A. M. Whelan: Theory of the octavic.

Eight numbers may be arranged into products of differences of the type $\overline{12} \cdot \overline{34} \cdot \overline{56} \cdot \overline{78}$ in 105 ways. These fall into sets of 
7 such that all 28 pairs occur. Limiting these sets to those of a certain symmetrical type we find that there are 30 such sets. A sign is attached to each product and the sum of each set of 7 products formed. These sums are the Coble heptads. These 30 heptads fall into 2 sets of 15 such that an even permutation among the 8 numbers leaves each set of 15 unaltered while an odd permutation sends one set into the other. In other words, each set of 15 heptads admits the group $\frac{1}{2} 8$ ! These sets are referred to as the $\sigma$ and $s$ heptads.

The following relations are readily proved: $\sum^{15} \sigma_{i}{ }^{n}$ $-\sum^{15} s_{i}{ }^{n}=0$ for $n=1,2,3,4,5,6,8 ; \sum^{15} \sigma_{i}^{7}-\sum^{15} s_{i}{ }^{7}$ $=\mu \sqrt{\Delta}$, where $\Delta$ is the discriminant of the octavic whose roots are the 8 quantities, and $\mu$ is a constant which is determined by consideration of a particular case. The sums of the powers of the 30 heptads are invariants of the octavic. Invariantive conditions for a triple root are obtained.

5. Dr. G. Y. Rainich: Analogy between electromagnetic field and analytic function.

The counterparts of the Cauchy-Riemann equations of Volterra's generalized theory of analytic functions coincide for $n=4$ with the electromagnetic equations for vacuum in Minkowsky form. The simplest singularity is a singular line which may be identified with an electron path. The analog of the residuum is the electric charge, and its invariance follows from the analog of Cauchy's integral theorem. There is likewise an analogy with the theory of surfaces: the energy tensor is derived from the electromagnetic tensor in the same way as the curvature from the second differential form. If we consider the curvature of the space as produced by the energy tensor, the additional members which arise in one of the sets of the Minkowsky equations by substituting absolute for ordinary differentiation make these equations non-linear in the components of the electromagnetic tensor (appreciable only near the singularities). This non-linearity may account for the equality of charges of all electrons, for the difference between positive and negative electricity, and for the dependence of the electron paths on the field.

6. Professor J. W. Alexander: The existence of closed geodesics on surfaces. Preliminary communication.

Associated with a surface $S^{2}$, there is a three-dimensional manifold $M^{3}$ such that a point of $M^{3}$ corresponds to a point 
of $S^{2}$ together with a fixed direction through the point. The geodesics of $S^{2}$ correspond to a congruence of curves of $M^{3}$, and every closed geodesic of $S^{2}$ corresponds to a closed curve of the congruence in $M^{3}$. The author proves that the sum of the indices of the closed curves of the congruence is a topological invariant of $M^{3}$. From this may be inferred a theorem by Birkhoff on the existence of a closed geodesic on any closed surface of genus 0 . The method is extended to the study of the closed geodesics of characteristic surfaces bounded by curves of zero velocity, such as arise in dynamical problems.

7. Mr. F. H. Murray: The asymptotic expansion of the functions $W_{k, m}(z)$ of Whittaker.

The asymptotic expansion of the functions $W_{k, m}(z)$ defined by Whittaker is already known, if $z$ lies in a sector which does not include the negative half of the real axis; it is shown here that this expansion continues to hold near the negative half of the real axis if the parameters $k, m$ satisfy a certain inequality. An application is made to the study of the "croissance" of the solutions of certain linear differential equations of the second order.

8. Professor Arnold Emch: Some geometric applications of symmetric substitution groups.

The geometry of the symmetric group in its fundamental aspects has been investigated by G. Veronese.* As the ultimate object of Veronese was a penetrating study of Pascal's hexagon inscribed in a conic, its generalizations, and of related problems, little attention was given to the various possible invariant curves and surfaces of such groups Since then the literature on problems of invariance of forms under certain groups has grown extensively.

In this paper, the author investigates a number of curves and surfaces which are invariant under the symmetric $G_{6}$ and $G_{24}$, associated with ternary and quaternary spaces respectively. The results obtained may perhaps be indicated by two of the theorems: All sextics of the $G_{6}$ which pass through 5 independent sextuples of the $G_{6}$ pass through a sixth common sextuple. All space sextics of the $G_{24}$ form a net; every sextic of the group lies on six cubic cones.

* Interprétation géométrique de la thćorie des substitutions de $n$ lettres particulièrement pour $n=3,4,5,6$, en relation avec les groupes de l'hexagramme mystique, Annali di Matematica, (2), vol. 11 (1882), pp. 93-236. 
9. Dr. J. L. Walsh: A property of Haar's system of orthogonal functions.

This note considers the problem of determining an infinite normal orthogonal system of functions $\varphi_{1}, \varphi_{2}, \varphi_{3}, \cdots$ so that, when $\varphi_{1}, \varphi_{2}, \cdots, \varphi_{n}$ are known, the function $\varphi_{n+1}$ shall be chosen as that normalized function which is orthogonal to $\varphi_{1}$, $\varphi_{2}, \cdots, \varphi_{n}$ and whose total fluctuation is least. If the functions $\varphi$ are required to vanish at the ends of the interval considered, the solution of the problem is precisely Haar's system.

10. Dr. C. A. Nelson: The Riemann adjoints of certain completely integrable systems.

Completely integrable systems of partial differential equations have been used by Wilczynski and others to investigate the projective differential properties of a surface. For a non-developable surface this system consists of two linear homogeneous equations of the second order in two independent variables. The study of developables requires a system of three equations, two of the preceding type and a third equation of the third order. The author determines all such systems whose adjoints, in the sense of Riemann, also form a completely integrable system. The surfaces whose characterizing systems of differential equations possess this property are of four types. The non-developables are $(a)$ nonruled surfaces whose invariants of lowest order vanish; $(b)$ ruled surfaces which have at least one straight line directrix; $(c)$ all quadrics. The developables are $(d)$ quadric cones.

\section{Professor Edward Kasner: Intermediate curvatures in} Riemann space.

The Riemann curvature of an $n$-space at a point $P$ depends upon the orientation of a plane or 2-flat through $P$. Schur proved that if the result is independent of orientation it will also be independent of $P$. There is then a spherical space. The Ricci curvature depends on a line, or the normal $(n-1)$ flat. If this is to be independent of orientation, the present author in an earlier paper has shown that it must also be independent of $P$, so the space must obey Einstein cosmological equations. He now defines a curvature depending on a $k$-flat, which generalizes the earlier concepts. Probably an analog of Schur's theorem is also valid here. 
12. Dr. G. A. Pfeiffer: Concerning the common boundary of two or more regions.

The author shows that the common boundary of two or more connected regions (sets of inner points) is a continuum, i.e., a closed connected point set. Further, he shows that if the number of regions is greater than two, then the common boundary is a non-decomposable continuum (i.e., a continuum which is not the sum of two proper sub-continua) or the sum of two non-decomposable continua.

13. Dr. Louis Weisner: Some theorems on insolvable groups.

The object of this paper is to convert certain well known theorems concerning substitution groups into theorems on abstract groups. Among the theorems thus obtained are the following: If a group $G$ contains a subgroup $H$ of index $n$ in $G$, such that no subgroup of $G$ which includes $H$ contains a subgroup invariant under $G$, then the order of every invariant subgroup of $G$ is a multiple of $n$; if $n$ is not a power of a prime, $G$ is insolvable. If a group $G$, which is the direct product of $G_{1}$ and $G_{2}$, contains a maximal subgroup $H$ of index $n$, which includes no invariant subgroup of $G$, and if $G_{1}$ and $G_{2}$ contain two subgroups of $H$ which are of index $n$ in $G_{1}$ and $G_{2}$ respectively, then $G_{1}$ and $G_{2}$ are simply isomorphic simple groups.

14. Professor I. J. Schwatt: The value of $\sum_{k=1}^{n} \sum_{\alpha=1}^{2 k}$ $\tan ^{p} \alpha \pi /(2 k+1), \sum_{k=1}^{\infty} \prod_{\alpha=1}^{2 k} \operatorname{ctn}^{p} \alpha \pi /(2 k+1)$, and similar forms in terms of Bernoulli and Eulerian numbers.

The author obtains formulas connecting the preceding expressions with the Bernoulli and Eulerian numbers.

15. Professor O. E. Glenn: On the reduction of differential parameters in terms of finite sets. Preliminary report.

Within a functional domain of rationality defined by derivatives of the function in the arbitrary transformations on the variables, and of a certain quadratic irrationality $\Delta$ formed from the latter, the infinitude of covariantive differential parameters, involving $n$th order derivatives, of a binary quantic, $F=(d f)^{m}=(d \varphi)^{m}$, is expressible rationally and integrally in terms of arbitrary absolute invariants together with a set of $\frac{1}{2}(n+1)(n+2)(m+1)+2$ differential parameters. This finite set is given explicitly by generalized linear invariant elements and iterated jacobian transvectants formed from such elements. 
The problem of complete systems in simpler domains is under consideration.

16. Dr. George Rutledge: A new form of Stirling's interpolation formula.

Stirling's interpolation formula is written in a new form, and the relation to the usual form is shown. This formula is a direct consequence of a general formula for the polynomial determined by a set of evenly spaced points (JourNAL of Mathematics and Physics, Massachusetts Institute of Technology, vol. 2 (1922), p. 47) and has many advantages in theoretical investigations.

17. Professor Joseph Lipka: On the angle between two curves in $V_{n}$.

We consider any two curves $c_{1}$ and $c_{2}$ in a general curved space of $n$ dimensions issuing from a point $P$ in the same direction, i.e., they have an element of arc $P Q=d s$ in common. If $d \omega$ is the angle between the curves $c_{1}$ and $c_{2}$ at the point $Q$, we find that $(d \omega / d s)^{2}=k_{1}{ }^{2}+k_{2}{ }^{2}-2 k_{1} k_{2} \cos \theta$, where $k_{1}$ and $k_{2}$ are the geodesic curvatures of $c_{1}$ and $c_{2}$ at $P$, and $\theta$ is the angle between the principal geodesic normals to $c_{1}$ and $c_{2}$ at $P$. If one of the curves is the geodesic through $P$ in the direction $P Q$, we have immediately $d \omega / d s=k$, an intrinsic definition of the geodesic curve at a point. Compare the author's paper Sulla curvatura geodetica delle linee appartenenti ad una varietà qualunque, RENDICONTI, ACCADEMIA DEI Lincei, vol. 31, May, 1922.

18. Mr. R. L. Wilder: On accessibility of the boundary of a domain.

In a paper presented at the April, 1922, meeting of the Society, the author showed that in the space constituted by a plane continuous curve, all points of the boundary of any domain are accessible if the boundary is connected im kleinen. Schoenflies has shown* that in ordinary euclidean space of two dimensions all points of the boundary of a plane connected domain are accessible if the boundary is a continuous curve. In the present paper it is shown that both of these results are particular cases of the following theorem: $D$ being either a domain with respect to a continuous curve or a plane con-

* Die Entwickelung der Lehre von den Punktmannigfaltigkeiten, JAHRESBericht der Vereinigung, Ergänzungsband II (1908), p. 215. 
nected domain in the ordinary sense, and $B$ its boundary, then in order that any point $x$ of $B$ be accessible from $D$ it is sufficient either that (1) there exist a circle $K$ with center at $x$, such that the set of all points of $B$ that lie within $K$ is a subset of a connected im kleinen subset $S-D$ (where $S$ is the set of all points in the space considered) or that (2) $x$ belong to no continuum of condensation of $B$.

19. Mr. L. H. Rice: Determinantal relations based on a matrix whose elements fall into two classes.

The author generalizes certain relations published by Muir in $1922,{ }^{*}$ one of which was based on a matrix in which the elements in a selected rectangular array were assigned to one class and the remaining elements to a second class, while another relation was based on a matrix in which the main diagonal elements were assigned to one class and the remaining elements of the matrix to a second class. Muir's proofs employed respectively Laplace's expansion and Cayley's. The present writer, by making use of a simpler mode of proof, has done away with all restrictions as to the possible distribution of the elements into two classes. The classes may be intermingled in any way, the assignment of an element to the first or second class being independent of that of any other element. Muir has also given a rule for the introduction of certain arbitrary quantities into the matrix; a second rule of the same general nature is given in the present paper.

The results are extended to $p$-way determinants.

20. Professor S. Kakeya: Maximum modulus of some expressions of limited analytic functions.

Continuing investigations previously published $\dagger$ the author shows that of all functions $\{f(x)\}$ which are, in the closed domain $|x| \leqq 1$, analytic and of absolute value not exceeding unity there is one and, except for a constant factor of unit absolute value, only one function $f_{0}(x)$ such that for $f=f_{0}$ the expression $\left|\sum_{r=1}^{r=m} \sum_{\substack{r=n_{r} \\ s=0}}^{s} c_{r s} f^{(s)}\left(\alpha_{n}\right)\right|$, where the $c_{r s}$ are given constants and the $\alpha_{r}$ are given constants of absolute value less than unity, assumes its least upper bound $M$. This function $f_{0}(x)$ is found to be a rational function which on the periphery of its unit circle is of constant absolute value unity.

R. G. D. Richardson, Secretary of the Society.

* Edinburgh Royal Society Proceedings, vol. 42, p. 342.

$\dagger$ TôHokU IMperial University Science Reports, vol. 6 (1917), p. 53. 